\title{
Maximization of Data Gathering in Clustered Wireless Sensor Networks
}

Tianqi Wang, Student Member, IEEE, Wendi Heinzelman, Senior Member, IEEE, and Alireza Seyedi, Member, IEEE

\begin{abstract}
In this paper, we investigate the maximization of the amount of gathered data in a clustered wireless sensor network (WSN). The amount of gathered data is maximized by (1) choosing the optimal transmit power, and (2) selecting the optimal cluster head. For problem (1), we find closed-form solutions for the optimal or near optimal transmit power of cluster members (CM). For problem (2), we propose a near optimal cluster head selection (CHS) algorithm. The communication burden and computational complexity of CHS only grow linearly with the size of the cluster. In the proposed algorithms, iterations have been avoided in order to significantly lower the complexity of the algorithms compared with traditional iteration-based numerical optimization algorithms, making these approaches suitable for use in energy-constrained wireless sensor networks. The optimization gain is shown to be significant.
\end{abstract}

\section{INTRODUCTION}

In the domain of wireless sensor networks, there has been much research effort aimed at efficiently utilizing the limited energy at the sensor nodes [1][2]. Numerous strategies have been investigated to improve the energy efficiency of WSNs, including power control, mobile data sink deployment, multiple data sink deployment, nonuniform initial energy assignment, and intelligent sensor and relay deployment [3].

As another option to improve the energy efficiency of WSNs, clustering protocols have been broadly adopted due to their effectiveness and simplicity. In clustered sensor networks, neighboring nodes are grouped as clusters. One of the nodes in a cluster is selected as the cluster head, and the remaining nodes are the cluster members. The cluster head is usually in charge of certain local coordinations, such as collecting data from the cluster members and communicating with other clusters and the data sink, while cluster members simply transmit data to the cluster head. The cluster head may be selected in a randomized manner, such as in HEED [4] or LEACH [5]. Such a randomized selection of the cluster head, combined with rotating the cluster head position, can effectively avoid the early drain of the energy of a particular node. However, it cannot guarantee the optimality of the selection. Transmit power is also a very important factor that influences the energy efficiency of WSNs from the physical layer (PHY) [6]. Power control techniques can be easily adopted in clustering topologies [7].

This work was supported in part by the National Science Foundation under grant \# ECS-0428157 and in part by a Young Investigator grant from the Office of Naval Research, \# N00014-05-1-0626.

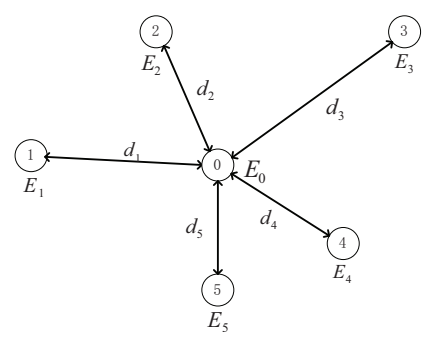

Fig. 1. A typical cluster topology $\left(N_{d}=5\right)$.

The energy efficiency of WSNs is usually evaluated by network lifetime. Network lifetime can be defined as the time elapsed until the first node in the network depletes its energy. This definition of lifetime can avoid the situation that certain nodes have very high power consumption, such as the common relay nodes of many routes, during network optimization. However, in a clustering-based network, due to the uneven importance of cluster members and cluster heads, the definition of network lifetime needs to be modified. In this paper, we define the lifetime of a cluster as the time duration for a cluster to function properly, i.e., the time elapsed until the cluster head dies or all cluster members die, whichever comes first. In data-centric applications, however, lifetime itself is not as meaningful a criterion as the amount of data gathered during the lifetime to evaluate the performance of a cluster. Thus, the goal of our proposed optimization is to maximize the amount of data gathered during the lifetime of a cluster, under energy and fairness constraints. The optimization parameters are the transmit power of the cluster members and the selection of the cluster head.

\section{TRANSMIT POWER OPTIMIZATION}

\section{A. Optimization Model}

Fig. 1 shows a typical cluster topology with 5 cluster members $(\mathrm{CMs})$ and 1 cluster head $(\mathrm{CH})$. In this paper, cluster members operate under a fairness constraint. The fairness constraint of a CM is defined as the constraint that the cluster head should collect an equal number of information bits from each CM. That is, if every CM $i$ works for time duration $T_{i}$, then during $T_{i}$, each CM transmits $D$ bits of data to the cluster head. This fairness constraint is due to the fact that the sensor nodes in a cluster are usually geographically close, and thus they observe the same phenomenon and generate the same amount of samples. Consequently, they generate the same amount of data for transmission given every node 
has the same source coding rate. We assume that a sufficient amount of data has been generated and stored in the source nodes' buffers before the communication procedure begins. Moreover, in this paper, a perfect time division multiple access (TDMA) scheduling is assumed. Therefore, no multiple access interference (MAI) is considered in this paper.

Given the fairness constraint and Shannon's channel capacity theorem, we have

$$
B T_{i} \log \left(1+\frac{P_{i}}{d_{i}^{n} P_{n, i}}\right) \geq D, \quad \forall i \in\left\{1,2, \ldots, N_{d}\right\},
$$

where $B$ is the bandwidth, $P_{i}$ is the transmit power of cluster member $i, d_{i}$ is the transmission distance from cluster member $i$ to the cluster head, $n$ is the path loss exponent, $P_{n, i}$ is the additive white gaussian noise (AWGN) power on the link from node $i$ to the cluster head, and $N_{d}$ denotes the number of cluster members.

Rearranging Eq. (1), the transmit power of $\mathrm{CM} i$ is

$$
P_{i} \geq\left(2^{\frac{D}{B T_{i}}}-1\right) d_{i}^{n} P_{n, i} .
$$

That is, the transmit power of CM $i$ is determined by its operation time duration $T_{i}$ and the total transmitted data $D$.

Moreover, the energy constraints at the CMs are

$$
T_{i}\left(P_{i}+P_{C T}\right) \leq E_{i}, \forall i \in\left\{1,2, \ldots, N_{d}\right\},
$$

where $P_{C T}$ denotes the circuit power consumption at the CMs, and the transmit powers are constrained by Eq. (2).

At the cluster head, the energy consumption comes from receiving the data from cluster members. Therefore, the energy constraint at the cluster head is

$$
P_{C R} \sum_{i=1}^{N_{d}} T_{i} \leq E_{0},
$$

where $P_{C R}$ is the circuit power consumption to receive data.

\section{B. Problem Formulation}

The resulting maximization of the total gathered data given the energy constraints and the fairness constraint can be formulated as

$$
\begin{array}{cl}
\min & -D \\
\text { s.t. } & C_{1}: T_{i}>0 \\
& C_{2}: P_{C R} \sum_{i=1}^{N_{d}} T_{i} \leq E_{0} \\
& C_{3}: T_{i}\left[P_{i}+P_{C T}\right] \leq E_{i}, \\
& C_{4}: P_{i} \geq\left(2^{\frac{D}{B T_{i}}}-1\right) d_{i}^{n} P_{n, i},
\end{array}
$$

where $i \in\left\{1,2, \ldots, N_{d}\right\}$. In this model, constraints $C_{2}$ and $C_{3}$ are the energy constraints at the cluster head and cluster members, respectively. $C_{4}$ results from the fairness constraint. Our goal is, for given residual energies and communication environments, to find the optimal cluster member operation time durations $\left[T_{1}, T_{2}, \ldots, T_{N_{d}}\right]$ and transmit powers $P_{i}$ that maximize the total data gathered at the cluster head.

Problem (5) is a minimum feasible set test problem with variables $T_{i}, P_{i}$ and $D$ [8]. Assume that the optimal solution is $D^{*}, T_{i}^{*}$ and $P_{i}^{*}$, where $P_{i}^{*}=\left(2^{D^{*} / B T_{i}^{*}}-1\right) d_{i}^{n} P_{n, i}+\epsilon_{i}, \epsilon_{i}>0$.
It is obvious that $D^{*}, T_{i}^{*}$ and $\hat{P}_{i}$, where $\hat{P}_{i}=\left(2^{D^{*}} / B T_{i}^{*}-\right.$ 1) $d_{i}^{n} P_{n, i}+\epsilon_{i} / 2, \epsilon_{i}>0$, is also an optimal solution, as $D^{*}$, $T_{i}^{*}$ and $\hat{P}_{i}$ satisfy the constraints. Therefore, we can always have the optimal transmit power $P_{i}^{*} \rightarrow\left(2^{D^{*} / B T_{i}^{*}}-1\right) d_{i}^{n} P_{n, i}$ from the right hand side. Thus, we can replace the inequality in constraint $C_{4}$ with equality. The resulting problem model is

$$
\begin{array}{cl}
\min & -D \\
\text { s.t. } & C_{1}: T_{i}>0, \\
& C_{2}: P_{C R} \sum_{i=1}^{N_{d}} T_{i} \leq E_{0} \\
& C_{3}: T_{i}\left[P_{i}+P_{C T}\right] \leq E_{i}, \\
& C_{4}: P_{i}=\left(2^{\frac{D}{B_{i}}}-1\right) d_{i}^{n} P_{n, i} .
\end{array}
$$

The optimal solutions of the above problem are arbitrarily close to the optimal solutions of problem (5). We determine an iteration-free solution to this problem in the next subsection.

\section{Transmit Power Optimization Algorithm}

Problem (6) is a typical minimum feasible set test of a quasiconvex optimization problem, which can be readily solved through iterative numerical methods [8]. However, performing hundreds of iterations is prohibitively complex in energylimited WSNs, and a simple solution with few or no iterations is desirable. In this section, we propose a simple iteration free solution to find the near optimal transmission time durations and transmit powers of each $\mathrm{CM}$ in a cluster.

When we fix the energy of the cluster members $\left(E_{i}\right)$ and increase the energy of the fusion center $\left(E_{0}\right)$ from zero to infinity, we observe that as $E_{0}$ increases, the maximum data gathered at the $\mathrm{CH}$ increases until it reaches a saturation limit. That is, there are two regions of maximum data gathered, $D^{*}$, as $E_{0}$ increases, namely the $\mathrm{CH}$-constrained region and the CM-constrained region. In the $\mathrm{CH}$-constrained region, the cluster head energy is the limiting factor in the total amount of data collected, while in the CM-constrained region, the residual energy at the CMs becomes the limiting factor. Our goal is to find an iteration-free solution for $T_{i}$ that maximizes $D$ in both the $\mathrm{CH}$-constrained and the $\mathrm{CM}$-constrained regions. After obtaining the optimal $T_{i}$, the corresponding optimal transmit power $P_{i}$ of the CMs can be easily determined.

1) CH-constrained region: In the $\mathrm{CH}$-constrained region, the limiting factor on the amount of data collected is the cluster head energy. In this case, the cluster head and all cluster members should use up their energy. Otherwise, any cluster member having energy left indicates that at least one of the cluster members limits the increase of the collected data and the cluster is operating in the CM-constrained region. Therefore, both the cluster head and the cluster member energy constraints are active. That is

$$
\begin{array}{r}
\sum_{i=1}^{N_{d}} T_{i}=\frac{E_{0}}{P_{C R}}, \\
T_{i}\left[\left(2^{\frac{D}{B T_{i}}}-1\right) d_{i}^{n} P_{n, i}+P_{C T}\right]=E_{i} .
\end{array}
$$


From (8), we have

$$
\begin{aligned}
& \log _{2}\left(\frac{\frac{E_{i}}{T_{i}}-P_{C T}}{d_{i}^{n} P_{n, i}}+1\right)=\frac{D}{B T_{i}}, \\
& \stackrel{a}{\Longrightarrow} T_{i} \log _{2}\left(\frac{E_{i}}{T_{i} d_{i}^{n} P_{n, i}}\right) \approx \frac{D}{B}, \\
& \Longrightarrow T_{i} \log _{2}\left(\frac{E_{i}}{d_{i}^{n} P_{n, i}}\right)-T_{i} \log _{2}\left(T_{i}\right) \approx \frac{D}{B}, \\
& \stackrel{b}{\Longrightarrow} T_{i} \approx \frac{D / B}{\log _{2}\left(\frac{E_{i}}{d_{i}^{n} P_{n, i}}\right)+\frac{6}{\ln 2}},
\end{aligned}
$$

where step $a$ follows by assuming that the signal-to-noise ratio is much greater than 1 and $E_{i} / T_{i} \gg P_{C T}$. Step $b$ follows from Borchardt's algorithm [9], which provides the following approximation that is the linearization of this function around zero: $T_{i} \log _{2}\left(T_{i}\right) \approx-6 T_{i} / \ln 2$.

Therefore, we have

$$
\begin{aligned}
\frac{T_{i}}{\sum_{i=1}^{N_{d}} T_{i}}= & \frac{\frac{D / B}{\log _{2}\left(\frac{E_{i}}{d_{i}^{n} P_{n, i}}\right)+\frac{6}{\ln 2}}}{D / B \sum_{i=1}^{N_{d}} \frac{1}{\log _{2}\left(\frac{E_{i}}{d_{i}^{n} P_{n, i}}\right)+\frac{6}{\ln 2}}} \\
= & \frac{1 /\left(\log _{2}\left(E_{i} / d_{i}^{n} P_{n, i}\right)+6 / \ln 2\right)}{\sum_{i=1}^{N_{d}} 1 /\left(\log _{2}\left(E_{i} / d_{i}^{n} P_{n, i}\right)+6 / \ln 2\right)} .
\end{aligned}
$$

Thus, from (7) and (10), we have

$$
T_{i}^{*} \approx \frac{E_{0}}{P_{C R}} \frac{1 /\left(\log _{2}\left(E_{i} / d_{i}^{n} P_{n, i}\right)+6 / \ln 2\right)}{\sum_{i=1}^{N_{d}} 1 /\left(\log _{2}\left(E_{i} / d_{i}^{n} P_{n, i}\right)+6 / \ln 2\right)} .
$$

The above approximation is accurate around zero. The optimal transmit power for node $i$ in the $\mathrm{CH}$-constrained region can be easily calculated by

$$
P_{i}^{*}=\frac{E_{i}}{T_{i}^{*}}-P_{C T}
$$

The maximum collected data from each node in the $\mathrm{CH}$ constrained region is then

$$
D^{*} \approx B T_{i}^{*} \log \left(1+\frac{P_{i}^{*}}{d_{i}^{n} P_{n, i}}\right) .
$$

2) CM-constrained region: In the $\mathrm{CM}$-constrained region, the maximum possible total data gathered from each cluster member as $E_{0} \rightarrow \infty$ is determined by the minimum value of the following sequence

$$
D^{*}=\min \left\{D_{1}, D_{2}, \ldots, D_{N_{d}}\right\},
$$

where the operator $\min \{\cdot\}$ returns the minimum element of a sequence, and $D_{i}$ are the values of the following unconstrained maximization problems:

$$
D_{i}=\max \left\{B T_{i} \log \left(1+\frac{\frac{E_{i}}{T_{i}}-P_{C T}}{d_{i}^{n} P_{n, i}}\right)\right\},
$$

The above unconstrained maximization problems are conducted over $T_{i}$, and their analytical solutions can be found as

$$
T_{i}^{\dagger}=\frac{E_{i}}{\frac{\ln 2\left(P_{C T}-d_{i}^{n} P_{n, i}\right)}{W\left(\frac{\ln 2}{2 d_{i}^{n} P_{n, i}}\left(P_{C T}-d_{i}^{n} P_{n, i}\right)\right)}+P_{C T}-d_{i}^{n} P_{n, i}},
$$

where $W(\cdot)$ is Lambert $\mathrm{W}$ function [10]. Although the Lambert $\mathrm{W}$ function can be calculated efficiently using numerical methods, it is still prohibitively complicated to calculate in sensor nodes. However, it is possible to further simplify (16) when $P_{C T} \gg d_{i}^{n} P_{n, i}$. In this case,

$$
T_{i}^{\dagger} \approx \frac{E_{i}}{\ln \left(\frac{\ln 2}{2 d_{i}^{n} P_{n, i}} P_{C T}\right)-3}+P_{C T} .
$$

Then

$$
D^{*}=\min \left\{B T_{i}^{\dagger} \log \left(1+\frac{\frac{E_{i}}{T_{i}^{\dagger}}-P_{C T}}{d_{i}^{n} P_{n, i}}\right)\right\},
$$

where exact, (16) or approximate, (17) values of $T_{i}^{\dagger}$ can be used.

Without loss of generality, assume $D_{1} \leq D_{i}, \forall i \in$ $\left\{2,3, \ldots, N_{d}\right\}$. Then we have

$$
D^{*}=D_{1}, T_{1}^{*}=T_{1}^{\dagger} .
$$

Moreover, $T_{i}^{*} \forall i \in\left\{2, \ldots, N_{d}\right\}$ can be any values that satisfy the following constraints:

$$
\begin{aligned}
\sum_{i=1}^{N_{d}} T_{i}^{*} & \leq \frac{E_{0}}{P_{C R}}, \\
T_{i}^{*}\left[\left(2^{\frac{D^{*}}{B T_{i}^{*}}}-1\right) d_{i}^{n} P_{n, i}+P_{C T}\right] & =E_{i} .
\end{aligned}
$$

Another important observation is that in the CM-constrained region, an increase in the cluster head energy cannot increase the total number of bits collected from the CMs. Thus, once the cluster enters the CM-constrained region, that is, when the energy at the $\mathrm{CH}, E_{0}$, is greater than a critical value, $\hat{E}_{0}$, an increase in the receiver energy becomes redundant. The value of $\hat{E}_{0}$ can be determined by the following equation

$$
\hat{E}_{0}=P_{C R} \sum_{i=1}^{N_{d}} T_{i}^{*}
$$

where $T_{1}^{*}=T_{1}^{\dagger}$ as defined by (19), and

$$
T_{i}^{*}=\min \left\{\arg \left[B T_{i} \log \left(1+\frac{\frac{E_{i}}{T_{i}}-P_{C T}}{d_{i}^{n} P_{n, i}}\right)=D^{*}\right]\right\},
$$

$\forall i \in\left\{2, \ldots, N_{d}\right\}$, where $D^{*}$ is from (18). Clearly the resulting $T_{i}^{*} \forall i \in\left\{2, \ldots, N_{d}\right\}$ also satisfy the constraint set (20).

Based on the previous analysis, the transmit power optimization algorithm that finds the near optimal solution to model (6) is summarized in Fig. 2. As shown in Fig. 2, the first step is to determine the condition of the cluster of interest ( $\mathrm{CH}$-constrained region or $\mathrm{CM}$-constrained region); once the operating condition is determined, the results from sections II-C1 and II-C2 can be used directly.

\section{Cluster Head Selection}

In this section, we propose a simplified method to identify the optimal cluster head, which only involves the calculation of (14) and (21) in each iteration. The simplified algorithm is based on a linear approximation around zero of the following functions: $D_{i}=B T_{i} \log \left(1+\left(E_{i} / T_{i}-P_{C T}\right) /\left(d_{i}^{n} P_{n, i}\right)\right)$. 


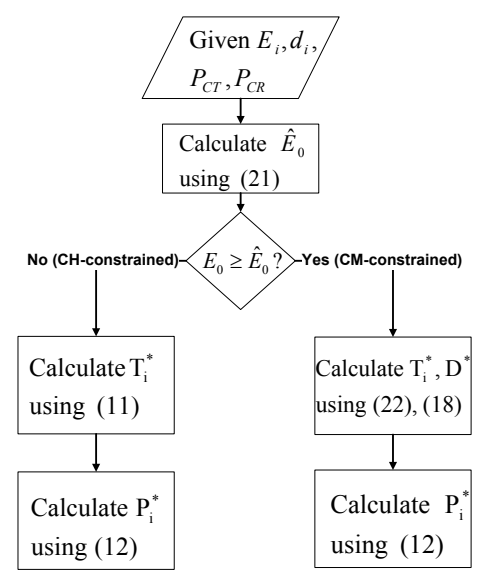

Fig. 2. The transmit power optimization algorithm flowchart.

First, we have the following observation: $D^{*}$ has a nearlinear relationship with the cluster head energy $E_{0}$ in the $\mathrm{CH}$ constrained region. The linearity is strengthened when $P_{C T} \gg$ $d_{i}^{n} P_{n, i}$ and $T_{i} \rightarrow 0$.

Proof: From (11), the optimal $T_{i} \mathrm{~s}$ and the energy of the cluster head $E_{0}$ has an approximately linear relationship in the $\mathrm{CH}$-constrained region. Therefore, to show that $D^{*}$ has a near-linear relationship with $E_{0}$ in the CH-constrained region, we only need to show the linearity of $D_{i}$ and $T_{i}$, for $i \in$ $\left\{1,2, \ldots, N_{d}\right\}$ :

$$
\begin{aligned}
& D_{i} \quad=B T_{i} \log \left(1+\frac{\frac{E_{i}}{T_{i}}-P_{C T}}{d_{i}^{n} P_{n, i}}\right) \\
& \Longrightarrow D_{i}=B T_{i}\left[\log \left(\frac{P_{C T}-d_{i}^{n} P_{n, i}}{d_{i}^{n} P_{n, i}}\right)\right. \\
&\left.+\log \left(-1+\frac{E_{i}}{\left(-d_{i}^{n} P_{n, i}+P_{C T}\right) T_{i}}\right)\right] \\
& \stackrel{a}{\Longrightarrow} \quad D_{i} \approx B T_{i}\left[\log \left(\frac{P_{C T}-d_{i}^{n} P_{n, i}}{d_{i}^{n} P_{n, i}}\right)+\log \left(\frac{E_{i}}{P_{C T}}\right)\right. \\
&\left.\quad-\log \left(T_{i}\right)+\log \left(1-\frac{P_{C T} T_{i}}{E_{i}}\right)\right] \\
& \stackrel{b}{\Longrightarrow} \quad D_{i} \approx B T_{i}\left[\log \left(\frac{P_{C T}-d_{i}^{n} P_{n, i}}{d_{i}^{n} P_{n, i}}\right)+\log \left(\frac{E_{i}}{P_{C T}}\right)+\frac{6}{\ln 2}\right] .
\end{aligned}
$$

The approximation $a$ becomes accurate when $P_{C T} \gg d_{i}^{n} P_{n, i}$, and the approximation $b$ becomes accurate when $T_{i} \rightarrow 0$. As shown by the above derivation, $D_{i}$ and $T_{i}$ have a near-linear relationship when $P_{C T} \gg d_{i}^{n} P_{n, i}$ and $T_{i} \rightarrow 0 . \square$

Let $D^{\prime}(i)$ denote the linear approximation of the maximum data collected for the cluster with node $i$ as the cluster head. The procedure for the proposed cluster head selection algorithm is straightforward: for each node $i$, calculate $D^{\prime}(i)$; then choose the node with the largest $D^{\prime}(i)$ as the cluster head.

In the proposed cluster head selection (CHS) algorithm, $D^{\prime}(i)$ can be expressed as

$$
D^{\prime}(i)= \begin{cases}D^{*} & E_{i} \geq \hat{E}_{0} \\ D^{*}-\frac{D^{*}}{\hat{E}_{0}}\left(\hat{E}_{0}-E_{i}\right) & E_{i}<\hat{E}_{0}\end{cases}
$$

where $D^{*}$ can be obtained from (14) and $\hat{E}_{0}$ can be calculated from (21).
The CHS algorithm can be distributed as follows (assuming each node knows the distances to its neighbors): first, each node uses one broadcast to inform the other nodes of its residual energy, and each node can then find its own $D^{\prime}$ through (23). The node with the most residual energy (assume it is node $i$ ) broadcasts to the other nodes to declare its $D^{\prime}(i)$. The rest of the nodes will compare the received $D^{\prime}(i)$ with their own $D^{\prime}(j), j \neq i$. If $D^{\prime}(j)>D^{\prime}(i)$, then node $j$ will notify node $i$ of its $D^{\prime}(j)$. Otherwise, node $j$ does not take any action. At last, node $i$ will compare the received information and use one broadcast to inform the rest of the nodes about the selected cluster head that provides the largest $D^{\prime}$. Note that the CHS algorithm only needs to be executed once at the beginning of the cluster formation.

\section{RESUlts}

In the transmit power optimization algorithm, the main result is the approximation made on the optimal operation time assignment $T_{i}$ through (11) in the CH-constrained region. Compared with the solutions of $T_{i}$ in the CM-constrained region (which are exact optimal solutions), (11) provides a near optimal approximation. Therefore, the effectiveness of the approximation needs to be evaluated. We assume that 5 sensor nodes are uniformly placed within a disk with a radius of $100 \mathrm{~m}$, centered at the cluster head. The path loss exponent is $n=4$. The circuit powers are $P_{C R}=30 \mathrm{~mW}$, and $P_{C T}=10$ $\mathrm{mW}$. The signal bandwidth is $B=100 \mathrm{KHz}$. The AWGN power is $-116.5 \mathrm{dBmW}$ and is equal on all links.

Fig. 3 shows the maximum data gathered when all CMs have random residual energy $E_{i}$ chosen from a uniform distribution between $1 \mathrm{~J}$ and $25 \mathrm{~J}$. The cluster head has energy $E_{0}$ varying from 0.01 to $0.5 \mathrm{~J}$. This setup guarantees that the cluster works in the $\mathrm{CH}$-constrained region. We compare four scenarios: (1) the optimal solution to problem (6) through numerical methods; (2) the proposed analytical approximation (iteration free solution); (3) each node has an equal transmission time duration constraint, with numerically optimized transmit power and time duration; (4) each node has an equal transmit power constraint, with numerically optimized transmit power and time duration.

The maximum amount of data collected is shown in Fig. 3. From Fig. 3, we can see that the proposed transmit power optimization algorithm provides a close approximation to the numerical optimization maximizing the amount of data collected in a $\mathrm{CH}$-constrained cluster. The proposed transmit power optimization algorithm achieves a significant gain compared to the cluster with equal power and equal transmission time duration constraints. For instance, when $E_{0}=0.3 \mathrm{~J}$, the cluster using the proposed transmit power optimization algorithm gathers an average of 1.2 times the maximum gathered data bits in the equal time duration case, and 8.9 times the number of the maximum gathered data bits in the equal transmit power case. Also shown in the figure is that the analytical approximation becomes more accurate as the cluster head energy decreases, which agrees with the analysis in section II. 
The performance of the proposed CHS algorithm is evaluated, and the results are shown in Fig. 4. The residual energy of the nodes is generated using a random variable $s$, which is uniformly distributed between 0 and 1 , through

$$
E_{i} \quad=2 s, \forall i \in\left\{0,1, \ldots, N_{d}\right\},
$$

where $N_{d} \in\{4,9,14,19\}$, and the nodes are placed within a disk with a radius $100 \mathrm{~m}$ following a uniform distribution.

In Fig. 4, the term linear approximation means that the proposed CHS algorithm by (23) is used to select the cluster head; while the term analytical approximation refers to a brute force cluster head selection approach, which uses the proposed transmit power optimization algorithm in each iteration, to select the optimal cluster head. The term Optimal refers to a brute force cluster head selection approach, which uses a numerical solution of model (6) through an interior-point method in each iteration. Moreover, the performance of a random selection and a worst case selection of the cluster head is provided. After cluster head selection, the proposed transmit power optimization algorithm is used to configure the transmit powers of the CMs in all cases.

Fig. 4 shows that the proposed CHS algorithm (linear approximation) provides a sound approximation to the optimal cluster head selection in terms of performance. That is, the maximum amount of data collected by the cluster using the CHS algorithm is almost as much as that of a cluster using the numerically optimized cluster selection, while the CHS algorithm avoids the iterations in the numerical optimization. Therefore, the proposed CHS algorithm has great potential in WSN applications. Also shown in Fig. 4, the analytical approximation method also provides a sound performance, although compared with the proposed CHS algorithm, it has slightly worse performance and higher complexity. The proposed CHS algorithm has a significant performance gain over the random selection and worst case selection. For example, when there are ten nodes in the cluster $\left(N_{d}=9\right)$, the cluster using the proposed CHS algorithm with linear approximation can collect 1.9 times the average number of maximum data bits collected by the clusters with the random selection, and 5.4 times the average number of maximum data bits collected by the clusters with the worst case selection.

\section{CONCLUSions}

In this paper, for transmit power optimization, we proposed an iteration-free algorithm for maximizing the amount of data gathered by a cluster throughout its lifetime, considering energy constraints and a strict data fairness constraint. Moreover, we developed a cluster head selection (CHS) algorithm to determine the optimal cluster head that provides the largest amount of collected data for a cluster. The performance gain by using the optimal transmit power and the optimal cluster head selection has been shown to be significant.

\section{REFERENCES}

[1] I. F. Akyildiz, W. Su, Y. Sankarasubramaniam, and E. Cayirci, "A Survey on Sensor Networks," IEEE Communications Magazine, pp. 102-114, Aug. 2002.

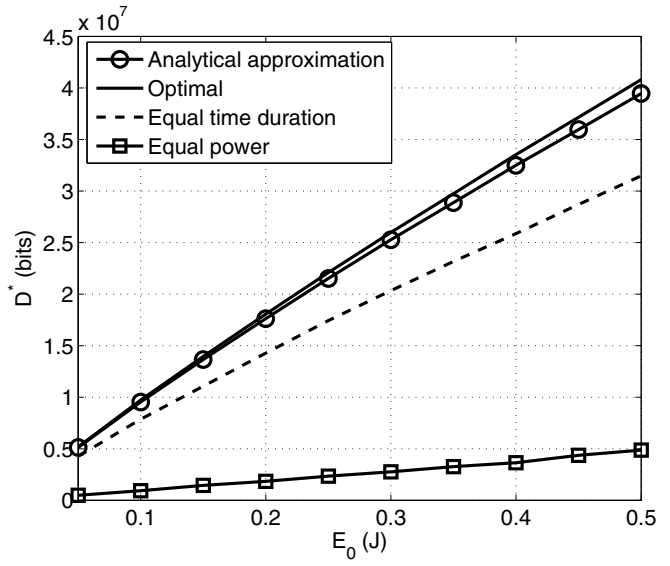

Fig. 3. The maximum data gathered by clusters operating in the $\mathrm{CH}-$ constrained region.

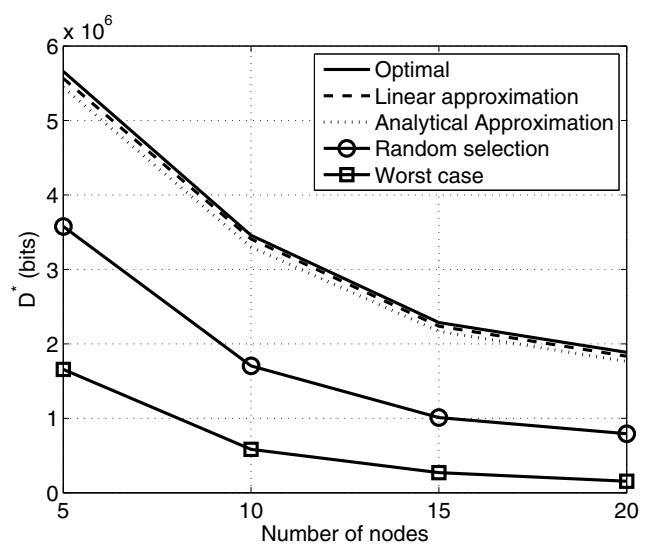

Fig. 4. The maximum data gathered by clusters with different cluster head selection strategies.

[2] A. J. Goldsmith and S. B. Wicker, "Design Challenges for EnergyConstrained Ad Hoc Wireless Networks," Wireless Communications, IEEE, Aug. 2002.

[3] C. Zhao, M. Perillo, and W. Heinzelman, "General Network Lifetime and Cost Models for Evaluating Sensor Network Deployment Strategies," IEEE Trans. on Mobile Computing, pp. 484-497, 2008.

[4] O. Younis and S. Fahmy, "Distributed clustering in ad-hoc sensor networks: A hybrid, energy-efficient approach," in Proceedings of the 23rd Annual Joint Conf. of the IEEE Computer and Communications Societies (INFOCOM), 2004.

[5] W. Heinzelman, A. Chandrakasan, and H. Balakrishnan, "An Application-Specific Protocol Architecture for Wireless Microsensor Networks," IEEE Trans. on Wireless Communications, pp. 660-670, Oct. 2002.

[6] C. S. Taek and A. Goldsmith, "Degrees of Freedom in Adaptive Modulation : A Unified View," in Proceedings of Vehicular Technology Conference, Spring, IEEE (VTS '01), 2001.

[7] S. Cui, A. Goldsmith, and A. Bahai, "Joint modulation and multiple access optimization under energy constraints," in Proceedings of Global Telecommunications Conference, IEEE (GLOBECOM '04), 2004.

[8] S. P. Boyd and L. Vandenberghe, Convex Optimization, 1st ed. Cambridge, UK: Cambridge University Press, 2004.

[9] R. W. Doerfler, Dead Reconing: Calculating without instruments. Houston: Gulf Publishing Company, 1993.

[10] E. M. Wright, "Solution of the Equation $z e^{z}=a$," Bull. Amer. Math. Soc., vol. 65, pp. 89-93, 1959. 